NASA Technical Memorandum 100810

AIAA-88-2377

\title{
A Semianalytical Technique for Sensitivity Analysis of Unsteady Aerodynamic Computations
}

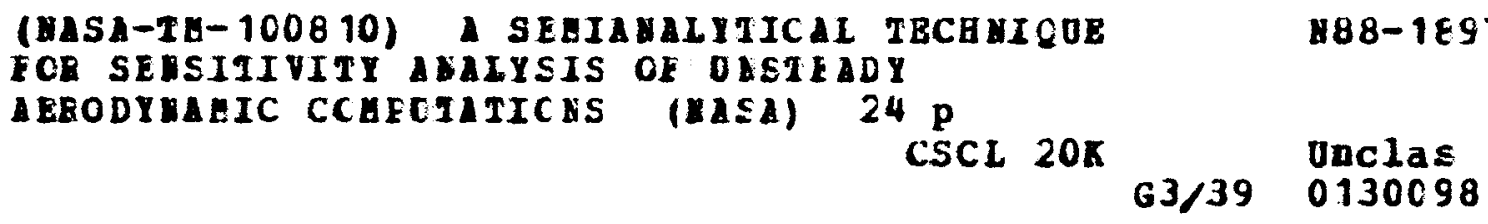

Durbha V. Murthy

University of Toledo

Toledo, Ohio

and

Krishna Rao V. Kaza

Lewis Research Center

Cleveland, Ohio

Prepared for the 29th Structures, Structural Dynamics and Materials Conference cosponsored by the AIAA, ASME, ASCE, AHS, and ACS Williamsburg, Virginia, April 18-20, 1988 
A SEMIANALYTICAL TECHNIQUE FOR SENSITIVITY ANALYSIS OF UNSTEADY

\title{
AERODYNAMIC COMPUTATIONS
}

\author{
Durbha V. Murthy* \\ University of Toledo \\ Toledo, Ohio 43606 \\ and \\ Krishna Rao V. Kaza** \\ National Aeronautics and Space Administration \\ Lewis Research Center \\ Cleveland, Ohio 44135
}

\begin{abstract}
SUMMARY
A semianalytical approach is developed for the sensitivity analysis of linear unsteady aerodynamic loads. The semianalytical approach is easier to implement than the analytical approach. It is also computationally less expensive than the finite difference approach when used with panel methods which require a large number of panels. The semianalytical approach is applied to an isolated airfoil in a two-dimensional flow and rotating propfan blades in three-dimensional flow. Sensitivity coefficients with respect to non-shapedependent variables are shown for some cases. It is expected that the semianalytical approach will be useful in aeroelastic design procedures particularly when mistuning is present and that it is potentially useful for shape sensitivity analysis of linear unsteady aerodynamics.
\end{abstract}

\section{INTRODUCTION}

The computation of derivatives of response quantities with respect to design parameters, known as sensitivity analysis, plays an important role in developing reliable and efficient procedures for design optimization of practical aerospace structures. Integrating the structural and aerodynamic design processes for developing better aerospace structures in an automated manner is gaining interest (ref. 1). Aerodynamic sensitivity analys is is necessary in order to make possible an efficient inter-disciplinary approach to the optimization of aerospace systems. However, the interest in sensitivity analysis has been mainly confined to structural applications (refs. 2 and 3 ). A recent paper (ref. 4), stressing the need for aerodynamic sensitivity analysis, contained only one reference on the subject and none dealing with unsteady aerodynamics. The present paper attempts to partially fill this void. Dwyer, Peterson and Brewer (refs. 5 and 6) applied sensitivity analys is to boundary layer flow equations to compute the effect of various parameters, though not in the context of design optimization. Recently, Haftka, et al. (ref. 7) presented a sensitivity analysis of steady aerodynamics for multidisciplinary

*NASA Resident Research Associate; Member AIAA and ASME.

**Senior Research Engineer; Associate Fellow, AIAA. 
optimization. The scarcity of published literature is perhaps due to the very complex nature of the aerodynamic response phenomena of practical aerospace structures. This is particularly true of rotary wing systems such as propfans. The prediction of steady and unsteady aerodynamic loads on these systems is still a subject of intense research. In addition, the coupling of computational aerodynamic codes with structural analysis and optimization programs is not straightforward.

The generalized unsteady aerodynamic loads on a structure are, in general, functions of the flow conditions, the structural geometry, and the structural motion. The derivatives of the generalized aerodynamic forces, with respect to the variables representing the structural geometry and motion, are useful in aeroelastic structural design procedures. Their computation constitutes the aerodynamic shape sensitivity analysis. Shape sensitivities are immensely useful in automated design of aircraft wings and rotating blades. For example, shape sensitivities can be used to compute optimal sweep distribution on propfan blades.

These sensitivity derivatives can be calculated using a straightforward finite-difference approach. However, experience in other engineering disciplines suggests that a semianalytical approach may be more computationally efficient. The objective of the present paper is to present a semianalytical approach for sensitivity analysis of subsonic unsteady aerodynamics used in flutter analysis, for the purpose of computing the derivatives of the generalized unsteady aerodynamic forces.

As a first step, only non-shape-dependent variables, representing the flow conditions and the structural motion, are considered. Such variables include free-stream Mach number, vibration frequency, mode shape and rotational speed. In addition to aeroelastic optimization, the derivatives with respect to flow conditions and structural motion can also be useful in computational schemes for aeroelastic analysis (refs. 8 to 10) and in computing the derivatives of the flutter Mach number and flutter frequency with respect to structural design variables (refs. 11 to 13).

In the following, the problem of numerical sensitivity analysis is introduced and the three possible approaches to the problem, (finite-difference, analytical, and the semianalyticall are described. The semianalytical approach is then described in further detail in the context of unsteady aerodynamic computations using panel methods. Finally, the application of sensitivity analysis to isolated airfoils in two-dimensional flow and to single rotation propfan blades in three-dimensional flow is presented along with some typical results.

NOMENCLATURE

A domain of integration

AN abbreviation for the analytical approach

bf parameter denoting coupling of first and second propfan normal modes

C matrix of aerodynamic influence coefficients 


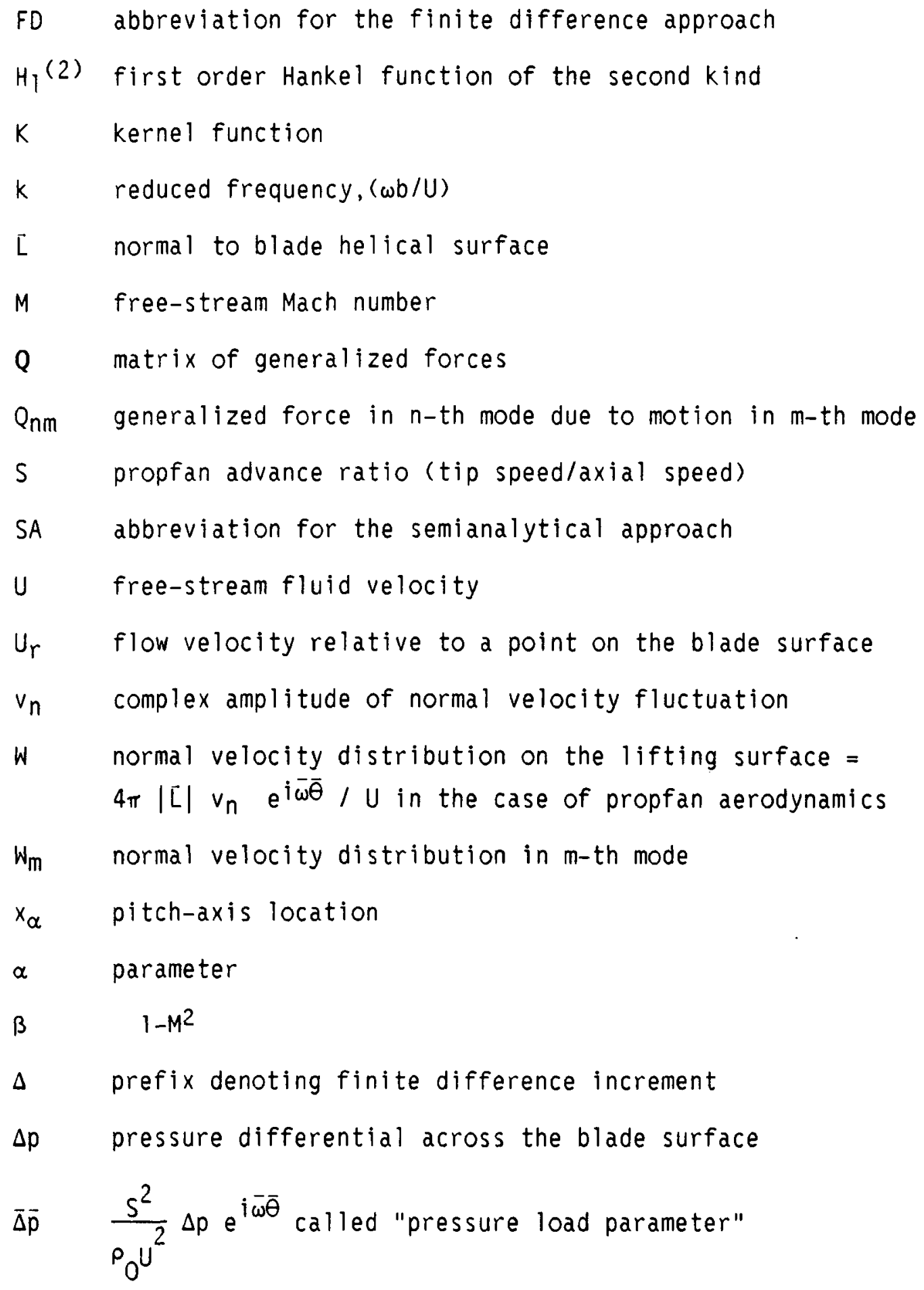

$\Delta p_{m} \quad$ pressure differential across the blade surface due to motion in $m$-th mode

$\delta_{n} \quad$ normal displacement in $n$-th mode

$\varepsilon \quad$ nondimensional control point location

$\bar{\theta}, \bar{\theta}_{0}$ angular coordinates 
$\xi \quad$ dimensionless chordwise pressure station coordinate

Po air density

$\tau \quad$ symbol for CPU times

$\Omega \quad$ propfan rotational speed

$\omega \quad$ blade vibration frequency

$\bar{\omega} \omega / \Omega$

- prime denotes variation

\section{NUMERICAL SENSITIVITY ANALYSIS}

In the finite difference approach, the sensitivity coefficient $f^{\prime}(x)$ of a function $f$ of a parameter $x$ is evaluated by repeating the analysis after incrementing each parameter. That is,

$$
f^{\prime}(x) \approx \varphi_{F D}(x)=\frac{f(x+\Delta x)-f(x)}{\Delta x}
$$

where the subscript FD denotes the finite difference approach and $\Delta x$ is the finite-difference step size. While only first order forward differences are considered, the comments apply equally to others. The finite difference approach is computationally expensive and accuracy is very much dependent on the selected step size $\Delta x$ and round-off error accumulation. The proper selection of a finite difference step size is not trivial. However, the finite difference approach is easy to implement.

The analytical approach to the problem of sensitivity analysis consists of evaluating the sensitivity coefficient by direct analytical differentiation. That is,

$$
f^{\prime}(x)=\varphi_{A N}(x)=\frac{d f}{d x}
$$

The subscript AN denotes the analytical approach. The analytical approach obviously results in the exact sensitivity coefficient. However, it is difficult to implement because the function $f(x)$ tends to be very complicated in practice and, in general, cannot be directly differentiated. Furthermore, many response functions of interest are computed by special purpose programs and analytical differentiation is impractical. In addition, in unsteady aerodynamic applications using panel methods, the cost of computing $\varphi_{A N}(x)$ is not much smaller than the cost of combuting $f(x)$. Thus the cost of their implementation is not offset by a substantial reduction in computational effort, as is often the case in structural applications. Hence, analytical methods of sensitivity analysis are not easily justified in unsteady aerodynamic applications.

The semianalytical approach consists of analytical differentiation of the original function with respect to an intermediate function, the derivative of 
which is then evaluated by numerical differentiation. Thus, if $f(x)=$ $f(g(x))$, then,

$$
f^{\prime}(x) \approx \varphi_{S A}(x)=\frac{d f}{d g} \cdot \frac{g(x+\Delta x)-g(x)}{\Delta x}
$$

The subscript SA denotes the semianalytical approach. The semianalytical approach combines the efficiency of the analytical approach with the ease of implementation of the finite difference approach. The most beneficial combination results if most of the computational effort of evaluating the function $f(x)$ is contained in computing $f(g)$ and most of the analytical complexity is contained in computing $g(x)$. It can also be sometimes expected to result in more accurate derivatives than the finite difference approach. For example, if $g(x)$ is nearly linear, then the $S A$ approach results in much more accurate derivatives than the FD approach.

\section{THE SEMIANALYTICAL APPROACH TO THE SENSITIVITY ANALYSIS OF UNSTEADY AERODYNAMICS}

The integral equation expressing the relation between the upwash and the pressure distribution can be written as (ref. 14)

$$
W=\int_{A} \Delta p K d A
$$

where $W$ represents the normal velocity distribution on the lifting surface, $\Delta p$ the distribution of the pressure differential between the upper and lower surfaces and $K$ the kernel function. $K$ may be formulated for steady, unsteady, two-dimensional, three-dimensional, incompressible, or compressible flow. Thus, the techniques are applicable to any of the above flow conditions, though the implementation presented herein is limited to two selected aerodynamic models. We consider unsteady compressible flow where structural motion is simple harmonic. The discussion, however, is also applicable to nonharmonic motion by replacing the real variable $\omega$, representing frequency, by the complex variable $s$, representing the Laplace transform variable. The normal velocity $W$ depends on the assumed motion of the lifting surface (structural motion) and is considered known. The kernel function for nonrotating systems depends on the flow Mach number and frequency of motion. For rotating systems, it also depends on the rotational speed. Given the coordinate position of the elemental area $\Delta A$, the kernel function can be computed at any point on the lifting surface. Knowing $W$ and $K$, the pressure distribution $\Delta p$ is obtained by inverting the integral equation above.

For aeroelastic applications, the primary interest lies not in the pressure distribution but in the generalized force contained in the matrix $Q$ given by

$$
Q_{n m}=\int_{A} \Delta p_{m} \delta_{n} d A
$$

where $\delta_{n}$ is the normal displacement in the $n$-th mode shape and $\Delta p_{m}$ is the pressure distribution resulting from motion in the m-th mode shape. If steady aerodynamic displacements are neglected, the normal displacement $\delta_{n}$ is independent of the assumed vibration frequency, $\omega$ and the flow conditions. 
We seek the derivatives of the generalized aerodynamic forces, contained in the matrix $Q$, with respect to parameters representing flow conditions and structural vibratory motion but independent of the domain of integration, $A$. Because the variation of generalized forces with respect to the mode shape is simpler, mode shape sensitivity is considered separately. First consider parameters that do not depend on the mode shape. Let $\alpha$ be one such parameter. Differentiating equation (5) with respect to $\alpha$,

$$
\frac{\partial Q_{n m}}{\partial \alpha}=\int_{A} \frac{\partial \Delta p_{m}}{\partial \alpha} \delta_{n} d A
$$

Thus, the sensitivity of the generalized force is equal to the generalized force acting on the blade resulting from a pressure differential across the blade surface given by $\partial \Delta p_{m} / \partial \alpha$. We refer to $\partial \Delta p_{m} / \partial \alpha$ as the "pseudo-pressure differential" corresponding to the parameter $\alpha$. to get

To obtain the pseudo-pressure differential, we differentiate equation (4)

$$
\frac{\partial W}{\partial \alpha}-\int_{A} \Delta p \frac{\partial K}{\partial \alpha} d A=\int_{A} \frac{\partial \Delta p}{\partial \alpha} K d A
$$

Equation (7) is an integral equation in the unknown $\partial \Delta p / \partial \alpha$ and is identical to equation (4) if the left hand side and $\partial \Delta p / \partial \alpha$ in equation (7) are replaced by $W$ and $\Delta p$ respectively. Thus, the sensitivity of the pressure distribution is equal to the pressure distribution that gives rise to the normal velocity distribution given by

$$
\frac{\partial W}{\partial \alpha}-\int_{A} \Delta p \frac{\partial K}{\partial \alpha} d A
$$

We refer to this velocity distribution as the "pseudo-upwash" distribution corresponding to the parameter $\alpha$. The pseudo-upwash is analogous to the concept of the pseudo-load used in the sensitivity analysis of static structural response (ref. 3).

To evaluate the pseudo-upwash, $\partial W / \partial \alpha$ and $\partial K / \partial \alpha$ must be computed. While it is often less difficult to evaluate $\partial W / \partial \alpha$ analytically, the analytical evaluation of $\partial K / \partial \alpha$ is generally time-consuming and difficult to implement. Therefore, the pseudo-upwash is evaluated by replacing the quantities $\partial W / \partial \alpha$ and $\partial K / \partial \alpha$ by their finite difference approximations, $\Delta W / \Delta \alpha$ and $\Delta K / \Delta \alpha$ respectively. Considering forward differences,

$$
\frac{\partial W}{\partial \alpha} \simeq \frac{\Delta W}{\Delta \alpha}=\frac{W(\alpha+\Delta \alpha)-W(\alpha)}{\Delta \alpha}
$$

and

$$
\frac{\partial K}{\partial \alpha} \simeq \frac{\Delta K}{\Delta \alpha}=\frac{K(\alpha+\Delta \alpha)-K(\alpha)}{\Delta \alpha}
$$

For sensitivity analysis with respect to mode shapes, we first note that the pressure distribution is a linear functional and the generalized force a 
quadratic functional of the mode shape. Hence, we consider variations instead of derivatives. Denoting variations by primes, we obtain from equation (5),

$$
Q_{n m}^{\prime}=\int_{A} \Delta p_{m}^{\prime} \delta_{n} d A+\int_{A} \Delta p_{m} \delta_{n}^{\prime} d A
$$

and from equation (4),

$$
W_{m}^{\prime}=\int_{A} \Delta p_{m}^{\prime} K d A
$$

so that, once again, the variation $\Delta p_{m}^{\prime}$ of the pressure distribution can be obtained by using $W_{m}$ as the pseudo-upwash distribution. Thus, for mode shape sensitivity analysis, we use equations (10) and (11) instead of equations (6) and (7) respectively.

For simple harmonic motion of an airfoil, the upwash can be approximated as (ref. 14)

$$
W_{m}(P)=U_{r} \frac{d \delta_{m}}{d s}(P)+i \omega \delta_{m}(P)
$$

so that

$$
W_{m}^{\prime}(P)=U_{r} \frac{d \delta_{m}^{\prime}}{d s}(P)+i \omega \delta_{m}^{\prime}(P)
$$

where $U_{r}$ is the flow velocity relative to the point $P$ on the airfoil and $s$ is the arc length along the blade surface at fixed span.

A popular way to solve the integral equation given by equation (4) for the pressure distribution is the family of panel methods. In these methods, the singular pressure distribution is approximated by a panelwise constantpressure distribution. The Kutta condition is implicitly satisfied by choosing specific control points. Panel methods can easily handle any planform and are most suitable for lifting surfaces of complex geometry. Fine paneling of the planform is necessary because of the rough approximation of panelwise constantpressure distribution.

Once a discretization scheme (such as a panel method) is devised, practically all of the analytical complexity in computing the generalized forces $Q_{n m}$ is contained in the computation of the kernel function $K$. Thus, a semianalytical sensitivity analysis scheme, using numerical differentiation for the kernel function derivative, is of considerable advantage in overcoming the implementation problems associated with the analytical approach. Also, the aerodynamic code used for analysis can be used for sensitivity analysis as well by simply replacing the real upwash distribution with the pseudo-upwash distribution and the real pressure differential with the pseudo-pressure differential. The approach adopted here is similar to the semianalytical approach popular in the sensitivity analysis of static structural response (refs. 15 and 16) and implemented in some general purpose finite element programs (ref. 17). 
The efficiency of the semianalytical approach in comparison to the finite difference approach depends on the size of the problem. The overall costs of computing the kernel function and of inverting the integral operator are roughly proportional to the square and the cube of the number of panels, respectively. Hence, if the number of panels is $n$, then the CPU time for the computation of the generalized forces can be expressed as

$$
\tau=\tau_{1} n^{3}+\tau_{2} n^{2}
$$

The first term represents the time required for inverting the integral operator and the second term the time required for kernel function evaluations. The semianalytical approach utilizes the same inverted integral operator (available from the solution of the corresponding analysis problem) for all derivatives, in contrast to the finite difference approach. Hence, the CPU times for the FD and the SA approaches of sensitivity analysis can be expressed as

$$
\begin{gathered}
\tau_{F D}=\tau_{1} n^{3}+\tau_{2} n^{2} \\
\tau_{S A}=\tau_{2} n^{2}
\end{gathered}
$$

In pressure formulations, for a moderate number of panels, the second term of equation (14), representing the kernel function computation dominates the overall computational time, so that $\tau_{F D}$ and $\tau_{S A}$ are of the same order. However, as $n$ becomes larger, the cost of the FD approach escalates more rapidly than that of the SA approach. Hence, when the number of panels is large, the efficiency gain of the SA approach over the FD approach can be substantial. A large number of panels is needed in the case of a complex geometry as that of a propfan, especially if mistuning is present (ref. 18). A further discussion of the computational cost of the solution of the integral equation is given by Clark and James (ref. 19).

\section{APPLICATIONS}

The semianalytical approach is applied to two unsteady aerodynamic models: (1) isolated airfoil in two-dimensional flov and (2) rotating propfan blades in three-dimensional flow. Sensitivity coefficients of generalized forces with respect to various parameters are calculated by both the FD and the SA approaches and compared. For the purpose of computing the finite difference derivatives, the step sizes were computed by a procedure described by Gill (ref. 20). This procedure seeks to minimize the total error (the sum of roundoff and truncation errors) in the sensitivity coefficient $f^{\prime}(x)$ using the round-off error expected in the evaluation of function $f(x)$. The round-of $f$ error in the evaluation of $f(x)$ is estimated by the difference in the values of $f(x)$ calculated in double precision and extended precision.

In the following, the logarithmic sensitivity function is defined as $x$ $f^{\prime}(x) / f(x)$. Thus, the sensitivity coefficient gives the absolute rate of change of the function $f(x)$, whereas the logarithmic sensitivity function gives a measure of the relative rate of change of $f(x)$. 
The kernel functed for this unsteady aerodynamic model is the Possio kernel (ref. 21) given by

$$
K(x, \xi)=-\frac{i M k}{8 \beta} e^{-i k(x-\xi)} \int_{-\infty}^{k(x-\xi) / \beta^{2}} e^{i \lambda} H_{1}{ }^{(2)}(M|\lambda|) \frac{d \lambda}{|\lambda|}
$$

where $M$ is the free-stream Mach number, $k$ the reduced frequency ( $\omega b / U)$, $\omega$ the blade oscillation frequency, $b$ the blade semi-chord, $U$ the free-stream flow velocity, and $x$ and $\xi$ represent the dimensionless chordwise control and pressure stations respectively. The Possio kernel is valid for subsonic Mach numbers. Bland (ref. 21) gives a full description of the computational aspects of the Possio kernel evaluation.

For the isolated airfoil, the parameters considered are the free-stream Mach number $M$, the reduced frequency $k$ and the location of the pitch axis $x_{\alpha}$. The nominal point chosen for this configuration corresponds to $M=0.8$, $k=0.10$, and $x_{\alpha}=-0.5$ (i.e., the pitch axis is at the quarter-chord). The downwash and pressure are computed at 32 stations along the chord. The finite difference step sizes were selected by minimizing the estimated total error in the sensitivity coefficients of selected normal velocities, pressure differentials and generalized forces. The estimated error in the derivatives (ref. 20) was less than 1.5 percent in all cases.

Figure 1 shows the variation of the generalized forces due to pitching motion and their sensitivity coefficients with respect to the parameter, $k$. Both the real parts (in phase with the motion) and the imaginary parts (in quadrature with the motion) are shown in figures $1(a)$ and (b) respectively. The axes on the right correspond to the function and those on the left to its sensitivity. The squares give the finite difference (FD) sensitivities and the crosses the semianalytical (SA) sensitivities. In all cases (except at zero reduced frequency), the FD and the SA coefficients were in very good agreement. It can also be seen that the variations of the generalized forces are consistent with the calculated values of the respective sensitivity coefficients. Similar results were obtained for generalized forces due to plunging and for sensitivities with respect to $M$ and $x_{\alpha}$.

Figure 2 shows the logarithmic sensitivities, defined in the previous section, of the generalized forces with respect to $k$. At this nominal point, the imaginary part of the lift due to pitching is seen to be the most sensitive to reduced frequency and the real parts of the lift due to pitching as well as plunging the least sensitive.

To compare the efficiency of the semianalytical method with the finitedifference method, the percentage saving of CPU time achieved by the semianalytical approach in figure 3 as a function of the number of chordwise stations. These CPU times were obtained in computing the sensitivity of generalized forces with respect to Mach number. The CPU time for the semianalytical approach ranges from $0.65 \mathrm{sec}$ when the number of chordwise stations is 32 to $63.7 \mathrm{sec}$ when the number is 256. The CPU times were obtained on the Amdahl 5860 computer using VS FORTRAN Version 2 compiler with no compiler optimization. 
PROPFAN BLADES IN THREE-DIMENSIONAL

COMPRESSIBLE FLOW

The second unsteady aerodynamic model studied is based on a threedimensional linear subsonic lifting surface theory in frequency domain and is developed by Williams and Hwang (ref. 22) for aeroelastic analysis of propfans (ref. 23).

The aerodynamic sensitivity analysis described below is implemented in a computer program called ASTROP3 at NASA Lewis Research Center. ASTROP 3 is an aeroelastic analysis program developed for aeroelastic analysis of single rotation propfans (ref. 23). An automated flutter search procedure (ref. 10), requiring the generalized force sensitivities, is also incorporated in ASTROP 3.

The integral equation expressing the relationship between the upwash and pressure distributions on a thin propfan blade is given by

$$
W(P)=-\int_{A} \bar{\Delta} \bar{p}\left(P_{0}\right) \frac{\partial}{\partial \bar{\theta}}\left[K\left(P, P_{0}\right)\right] d A_{0}
$$

Note that the definition of the kernel function does not exactly correspond to that of the previous section.

Williams and Hwang (ref. 22) discretized the above integral equation by splitting the blade into $n$ rectangular panels within each of which $\bar{\Delta} \bar{p}$ is assumed constant. One control point is assigned to each panel at the midspan and $100 \varepsilon$ percent chord position. The best value for $\varepsilon$ was empirically found by Williams and Hwang (ref. 22) to be 0.85 . The discretization results in the algebraic system of equations given by

$$
W=C \bar{\Delta} \bar{p} \quad \text { Or }, \quad \bar{\Delta} \bar{p}=C^{-1} W
$$

where $W$ is a vector of the values of $W$ at chosen control points on each of the panels, $\bar{\Delta} \bar{p}$ is a vector of the values of $\bar{\Delta} \bar{p}$ on each of the panels, and $C$ is a matrix of aerodynamic influence coefficients given by

$$
c_{i j}=-\int_{A_{j}} \frac{\partial}{\partial \bar{\theta}}\left[K\left(P_{i}, P_{0}\right)\right] d A_{0}
$$

where the subscripts $i$ and $j$ refer to the panel numbers.

The computation of the pseudo-pressure differential requires the derivative of the vector of the pressure load parameters, $\partial \Delta p / \partial \alpha . \partial \Delta p / \partial \alpha$ is obtained by differentiating equation (19),

$$
\frac{\partial \boldsymbol{W}}{\partial \alpha}=C \frac{\partial \bar{\Delta} \bar{p}}{\partial \alpha}+\frac{\partial C}{\partial \alpha} \bar{\Delta} \bar{p}
$$

or,

$$
\frac{\partial \bar{\Delta} \bar{p}}{\partial \alpha}=C^{-1}\left[\frac{\partial W}{\partial \alpha}-\frac{\partial C}{\partial \alpha} \bar{\Delta} \bar{p}\right]
$$

For mode-shape-dependent parameters, 


$$
\frac{\partial \bar{\Delta} \overline{\mathbf{p}}}{\partial \alpha}=\mathrm{C}^{-1} \frac{\partial \mathbf{W}}{\partial \alpha}
$$

because the aerodynamic influence coefficients are independent of mode shapes.

For propfan blades, the parameters considered are the free-stream Mach number $M$, the blade vibration frequency $\omega$, the rotational speed $\Omega$, the location of the control point $\varepsilon$ and a parameter bf denoting the coupling of the first and second normal modes of the blade. The parameter $b f$ defines the normal displacement of the blade mode shape as $\delta(P)=b_{f} \delta_{1}(P)+\left(1-b_{f}\right) \delta_{2}(P)$ where $\delta_{1}$ and $\delta_{2}$ are the normal displacements due to motion in the first and the second blade normal modes respectively. Thus, the parameter bf represents the coupling of the first two normal modes. In this formulation, the domain of integration $A$ is dependent on the advance ratio. In order not to change the shape of the helical surface and thus maintain the non-shapedependent nature of the variables, the advance ratio was artificially held constant while computing the derivatives with respect to the Mach number and the rotational speed. This limitation will be removed when the sensitivity analysis is extended to shape-dependent variables. The control point location $\varepsilon$ is also considered a parameter because its value is chosen empirically and the sensitivity of the generalized forces to $\varepsilon$ is of interest.

The planform and the normal modes of the SR3C-X2 propfan blade (ref. 23), shown in figure 4 , were used as an example. The normal modes analysis was performed using COSMIC NASTRAN considering centrifugal deformations but not steady aerodynamic deformations. The planform was divided into 72 panels. The finite difference step sizes were selected by minimizing the estimated total error in the sensitivity coefficients of selected normal velocities, influence coefficients, pressure differentials and generalized forces. The estimated error in the derivatives (ref. 20) was less than 2.4 percent in all cases.

\section{Sensitivity To Frequency, Mach Number and Rotational Speed}

The computed sensitivities with respect to the vibration frequency of the generalized forces due to motion in the first mode are shown in figure 5 along with the generalized forces on the right vertical axis. Figures 6 and 7 similarly show the sensitivities with respect to Mach number and the rotational speed respectively. Once again, the FD and the $S A$ sensitivities are in reasonable agreement. Both the FD and the SA sensitivities are sometimes affected by round-off error as evidenced by their wavy nature. This indicates that the step-size selection algorithm used (ref. 20) may be inadequate in some cases and an improved algorithm (ref. 24) should perhaps be employed.

\section{Sensitivity to Empirical Parameters}

An important application of sensitivity analysis is in judging the sensitivity of generalized forces to empirical parameters. Such sensitivity gives a measure of the confidence in the values selected for the empirical parameters. As an example of such an application, the sensitivities of the generalized forces are calculated with respect to the location $\varepsilon$ of the control point on the panel at which the normal velocity due to structural motion is specified. Figure 8 shows the logarithmic sensitivity of generalized forces 
with respect to the control point location. It is observed that the generalized force could be expected to change by up to 3 percent for a 1 percent change in the control point location. This is considered satisfactory.

Finally, for the 72 panels used here, the percent reduction in CPU time from the semianalytical approach over the finite difference approach was 8 to 13 percent. Greater reductions are expected for larger numbers of panels.

\section{CONCLUDING REMARKS}

A semianalytical approach to sensitivity analysis of linear unsteady aerodynamics is presented. Applications to isolated airfoils and propfan blades have shown that the semianalytical approach can be implemented relatively easily and that the semianalytical approach does not suffer from any accuracy problems compared to the finite difference approach. Preliminary studies show that the semianalytical approach will result in substantial savings in computational time for sensitivity analysis when the number of panels in propfan aeroelastic analysis is large. This is typically the case when mistuning is considered.

It is expected that the semianalytical approach will be useful for computing shape sensitivity derivatives of generalized unsteady aerodynamic forces. These derivatives are expected to be used in developing efficient analytical and design optimization procedures for complex aeroelastic structures.

\section{ACKNOWLEDGMENTS}

The authors acknowledge the assistance provided by Dr. S.R. Bland of NASA Langley Research Center and Dr. M.H. Williams of Purdue University.

\section{REFERENCES}

1. Grossman, B., Gurdal, Z., Haftka, R.T., Strauch, G.J., and Eppard, W.M., "Integrated Aerodynamic/Structural Design of a Sailplane Wing," AIAA Paper $86-2623$, Oct. 1986.

2. Haug, E.J., Choi, K.K., and Komkov, V., Design Sensitivity Analysis of Structural Systems, Academic Press Inc, Orlando, 1986.

3. Haftka, R.T. and Kamat, M.P., Elements of Structural Optimization, Martinus Nijhoff Publishers, Boston, 1985.

4. Sobieszczanski-Sobieski, J., "The Case for Aerodynamic Sensitivity Analysis," Sensitivity Analys is in Engineering, NASA CP-2457, 1986, pp. 77-96.

5. Dwyer, H.A., Peterson, T., and Brewer, J., "Sensitivity Analys is Applied to Boundary Layer Flow," Proceedings of the 5 th International Conference on Numerical Methods in Fluid Dynamics, A.I. Van de Vooren and P.J. Zandbergen, eds., Springer-Verlag, New York, 1976, pp. 179-184. 
6. Dwyer, H.A. and Peterson, T., "A Study of Turbulent Flow with Sensitivity Analysis," AIAA Paper 80-1397, July 1980.

7. Haftka, R.T., Grossman, B., Eppard, W.M., and Kao, P.J., "Efficient Optimization of Integrated Aerodynamic-Structural Design," presented at the International Conference on Inverse Design Concepts and Optimization in Engineering Sciences - II, University Park, PA, 1987.

8. Rudisill, C.S. and Cooper, J.L., "An Automated Procedure for Determining the Flutter Velocity," Journal of Aircraft, Vol. 10, No. 7, July 1973, pp. 442-444.

9. Bhatia, K.G., "An Automated Method for Determining the Flutter Velocity and the Matched Point," Journal of Aircraft, Vol. 11, No. 1, Jan. 1974, pp. $21-27$.

10. Murthy, D.V. and Kaza, K.R.V., "A Computational Procedure for Automated Flutter Analysis," NASA TM-100171, 1987.

11. Rudisill, C.S. and Bhatia, K.G., "Optimization of Complex Structures to Satisfy Flutter Requirements," AIAA Journal, Vol.9, No. 8, Aug. 1971, pp. 1487-1491.

12. Rao, S.S., "Rates of Change of Flutter Mach Number and Flutter Frequency," AIAA Journal, Vol. 10, No. 11, Nov. 1972, pp. 1526-1528.

13. Bindolino, G. and Mantegazza, P., "Aeroelastic Derivatives as a Sensitivity Analysis of Nonlinear Equations," AIAA Journal, Vol. 25, No. 8 , Aug. 1987, pp. 1145-1146.

14. Bisplinghoff, R.L. and Ashley, H., Principles of Aeroelasticity, Dover Publications, New York, 1975.

15. Camarda, C.J. and Adelman, H.M., "Static and Dynamic Structural Sensitivity Derivative Calculations in the Finite-Element-Based Engineering Analysis Language (EAL) System," NASA TM-85743, 1984.

16. Chengdong, $C$. and Yingwei, L., "New Computation Scheme of Sensitivity Analysis," presented at the First World Congress on Computational Mechanics, Austin, TX, Sept. 22-26, 1986.

17. Nagendra, G.K. and Fleury, C., "Sensitivity and Optimization of Composite Structures Using MSC/NASTRAN," Sensitivity Analys is in Engineering. NASA CP-2457, 1986, pp. 147-166.

18. Kaza, K.R.V., Mehmed, O., Moss, L.A., and Williams, M., "Analytical and Experimental Investigation of Mistuning on Propfan Flutter, "28th Structures, Structural Dynamics and Materials Conference, Part 2A, AIAA, New York, 1987, pp. 98-110. (NASA TM-88959).

19. Clark, R.W. and James, R.M., "New Approach to the Solution of Large, Full Matrix Equations," AIAA Journal, Vol. 19, No. 2, Feb. 1981, pp. 184-190. 
20. Gill, P.E., Murray, W., Saunders, M.A., and Wright, M.H., "Computing Forward-Difference Intervals for Numerical Optimization," SIAM Journal on Scientific and Statistical Computing, Vol. 4, No. 2, 1983, pp. 310-321.

21. Bland, S.R., "Development of Low-Frequency Kernel-Function Aerodynamics for Comparison with Time-Dependent Finite Difference Methods, "NASA TM-83283, 1982.

22. Williams, M.H. and Hwang, C.C., "Three Dimensional Unsteady Aerodynamics and Aeroelastic Response of Advanced Turboprops," 27th Structures, Structural Dynamics and Materials Conference, Part 2, AIAA NewTork, 1986. pp. $116-124$.

23. Kaza, K.R.V., Mehmed, O., Narayanan, G.V., and Murthy, D.V., "Analytical Flutter Investigation of a Composite Propfan Mode1," 28th Structures, Structural Dynamics and Materials Conference, Part 2A, AIAA, New York, 1987, pp. 84-97. (NASA TM-88944).

24. Iott, J., Haftka, R.T., and Adelman, H.M., "Selecting Step Sizes in Sensitivity Analysis by Finite Differences," NASA TM-86382, 1985. 

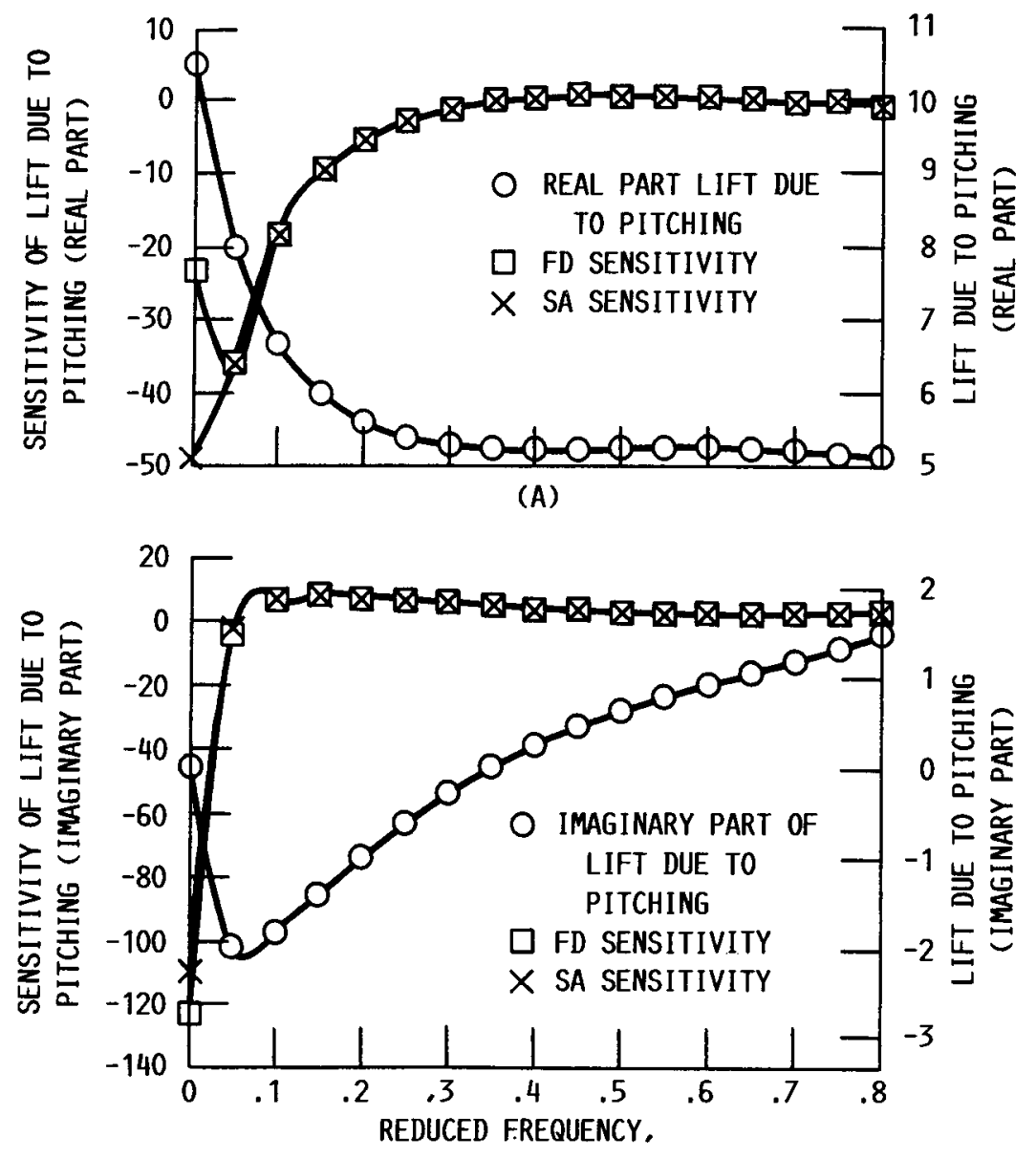

(B)

FIGURE 1. - SENSITIVITY OF GENERALIZED FORCES

TO FREQUENCY. 

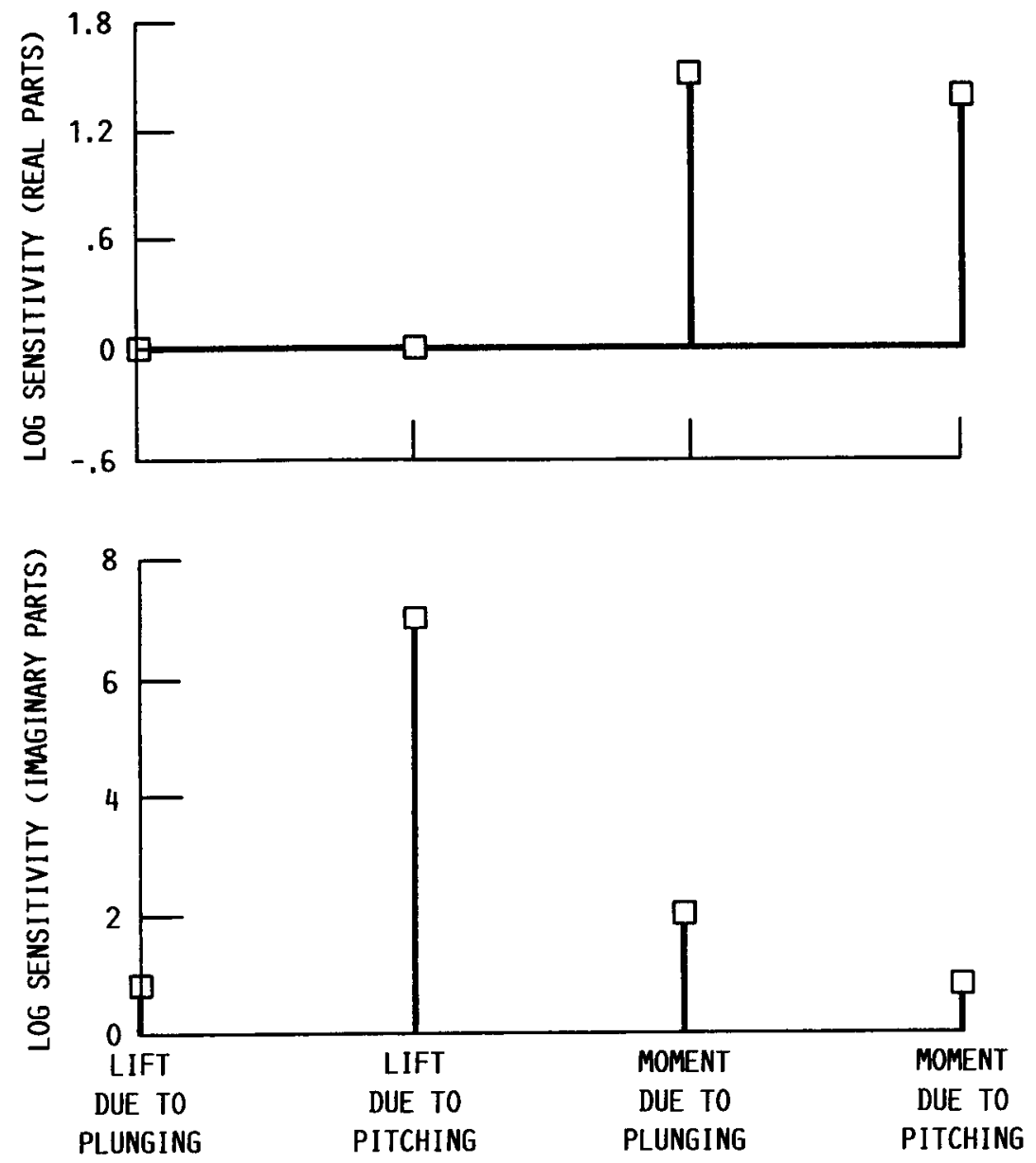

FIGURE 2. - SENSITIVITY OF GENERALIZED FORCES TO FREQUENCY. 


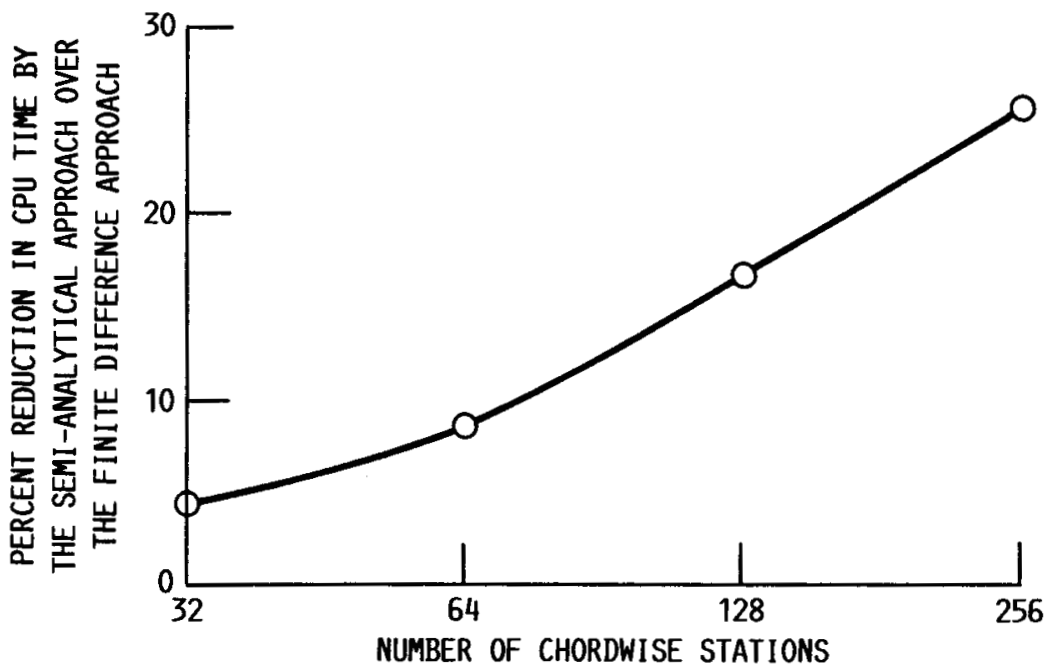

FIGURE 3. - EFFICIENCY OF THE SEMI-ANALYTICAL APPROACH OVER THE FINITE-DIFFERENCE APPROACH. 
ORIGINAL PAGE IS

OF POOR QUALITY

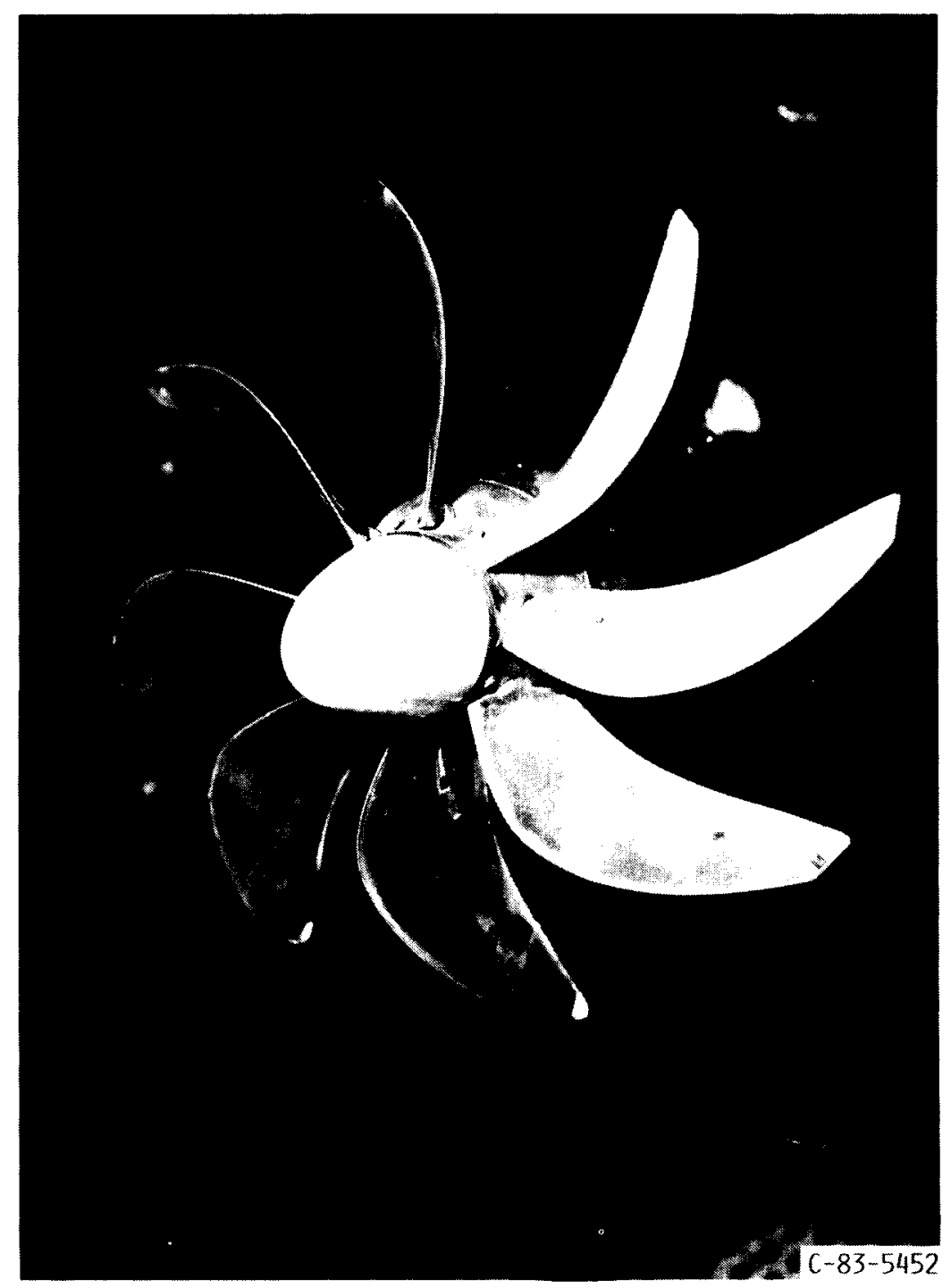

FIGURE 4. - THE SR3C-X2 PROPFAN MODEL IN THE LEWIS $8 \times 6$ FOOT WIND TUNNEL. 


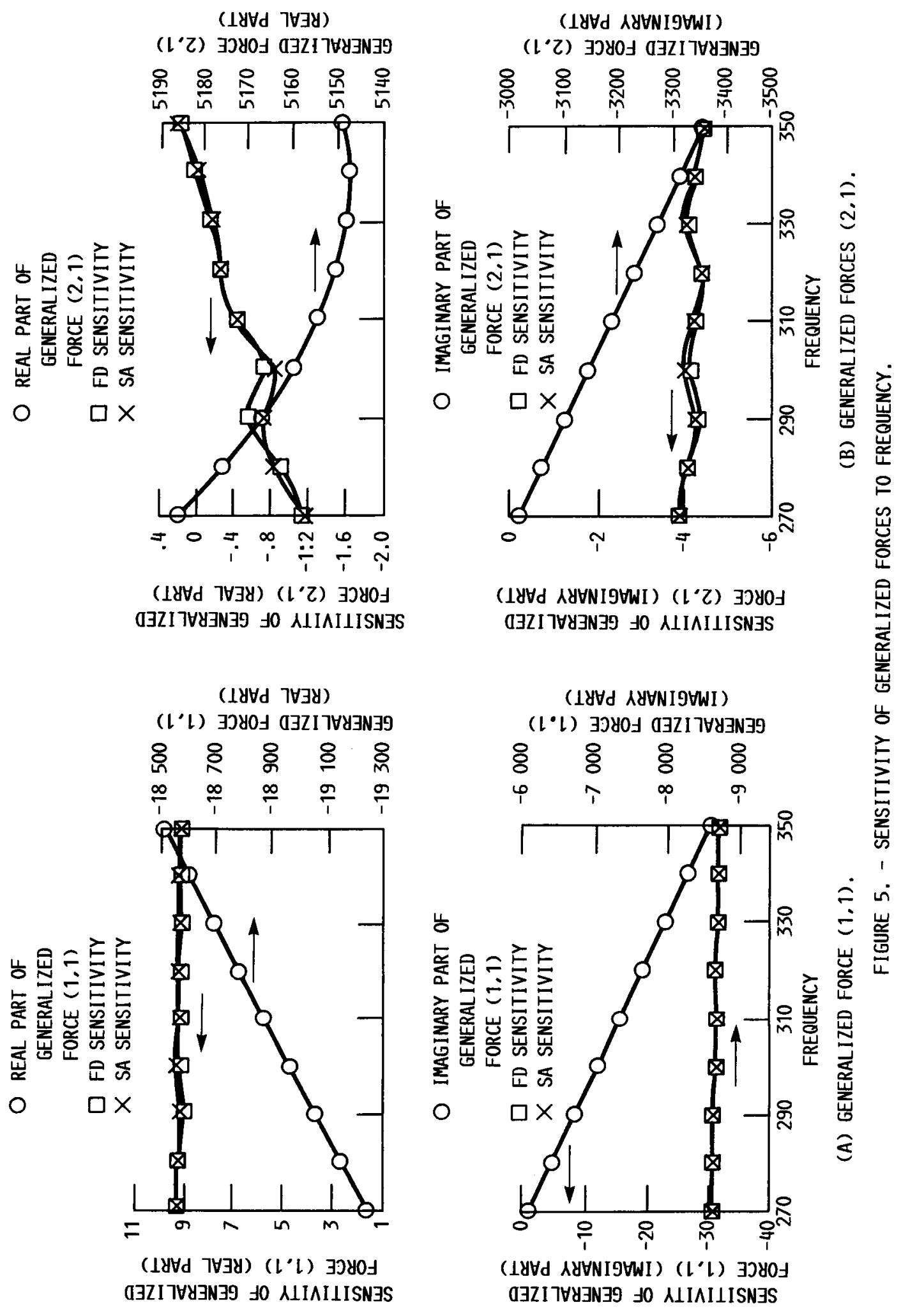


( $18 \forall d 7 \forall \exists y)$

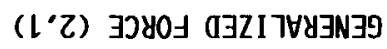

ర్రి

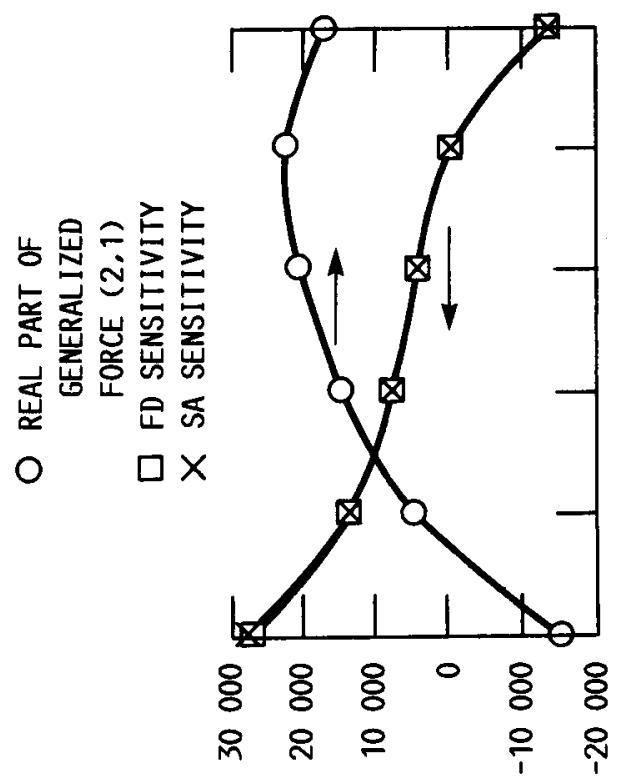

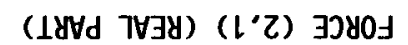

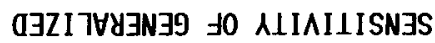

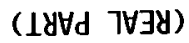

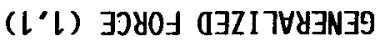

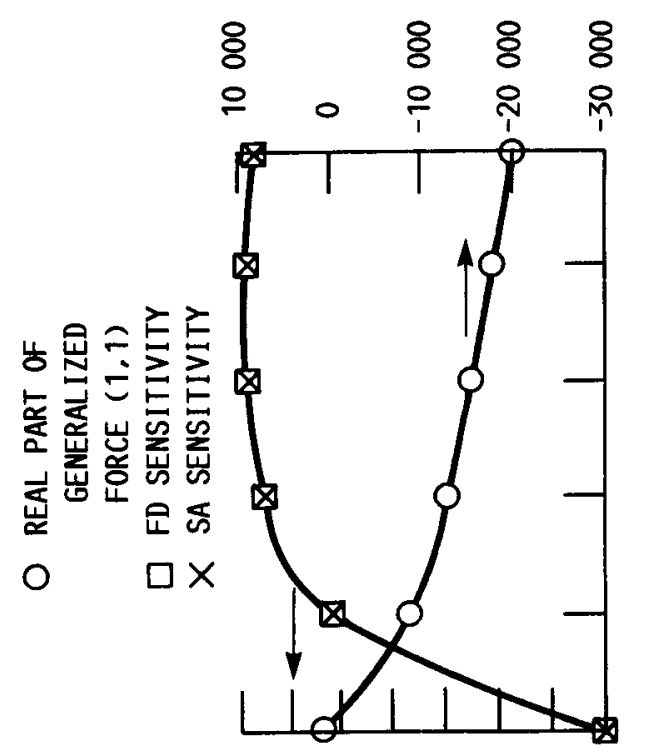

\& \& \& \& \& \& \& \&

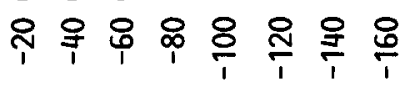

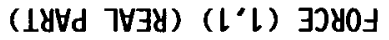

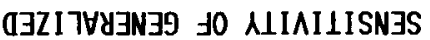

(IYYd NYVNIOWWI)

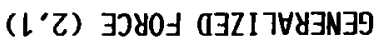

ষ্요 \& \& \&

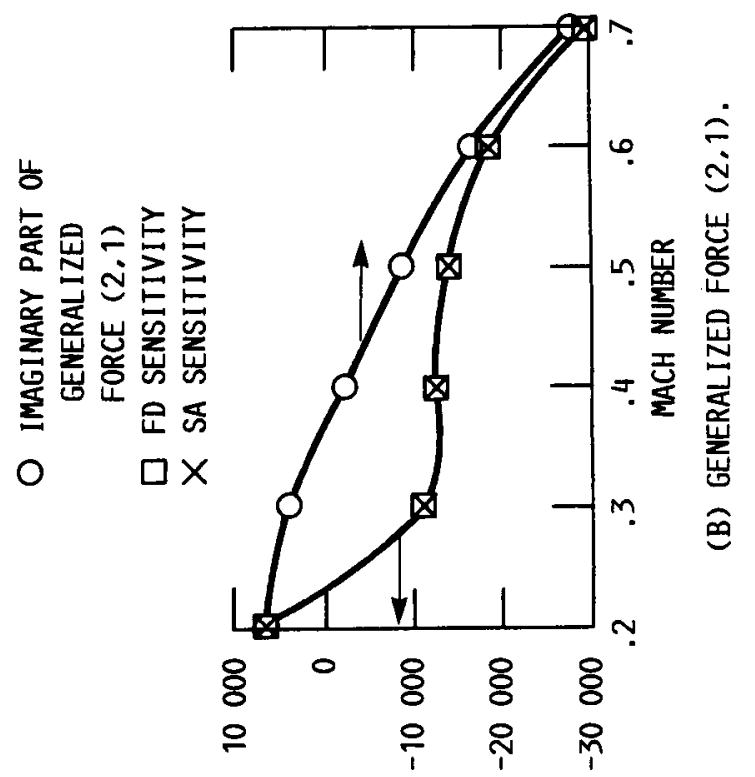

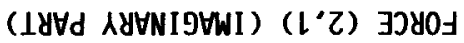

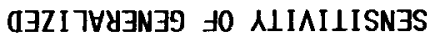

(IY甘d ᄉQ甘NIDHWI)

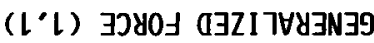

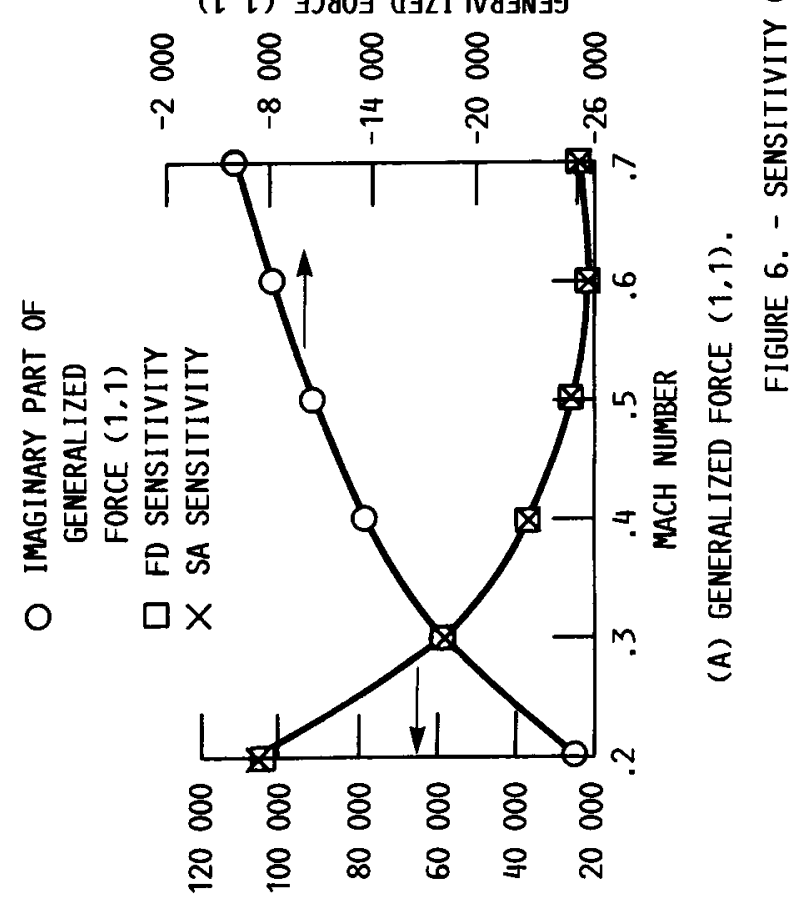

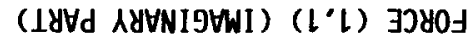

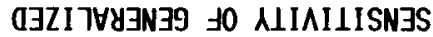



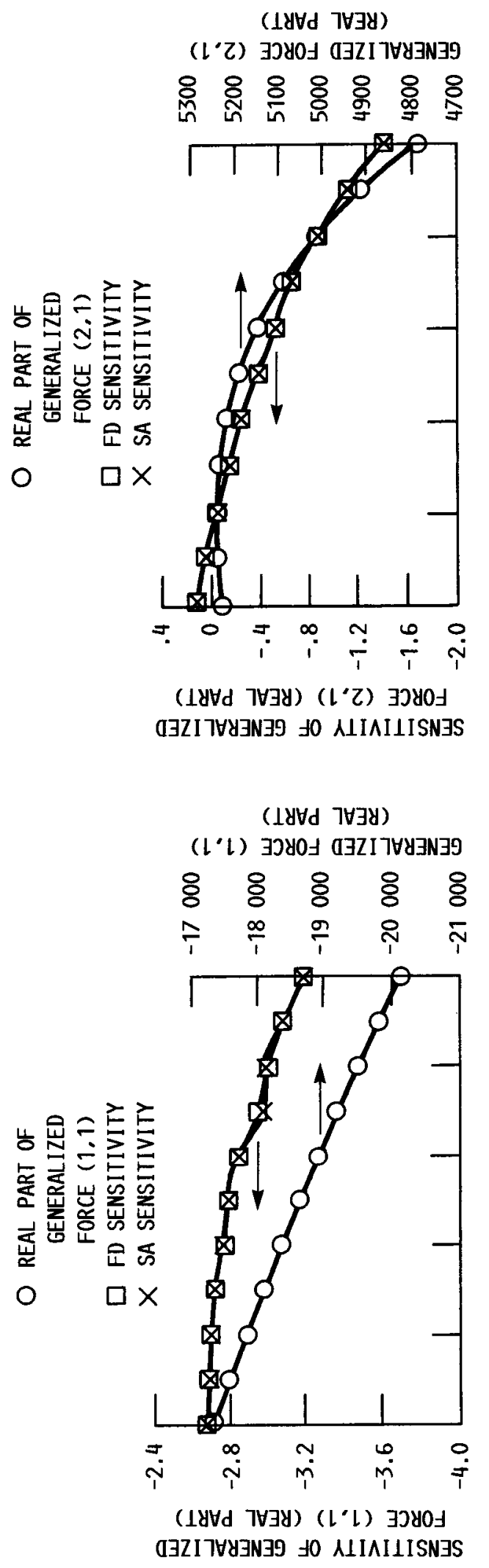

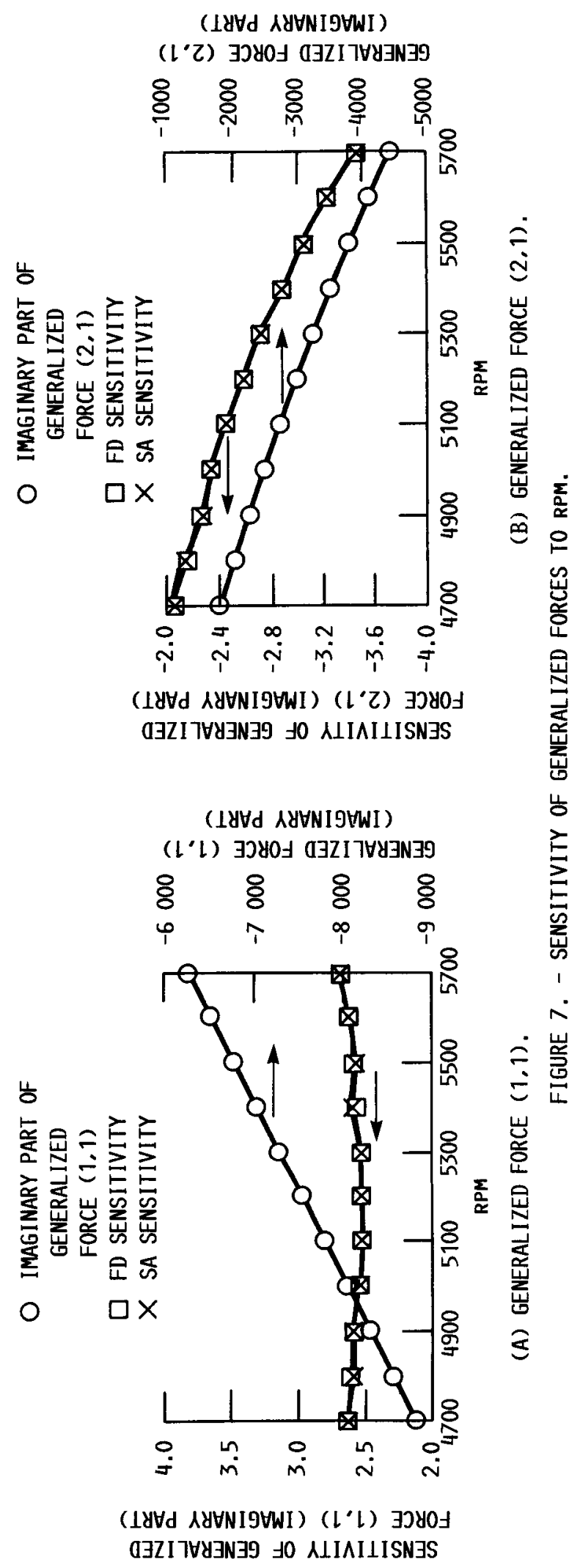



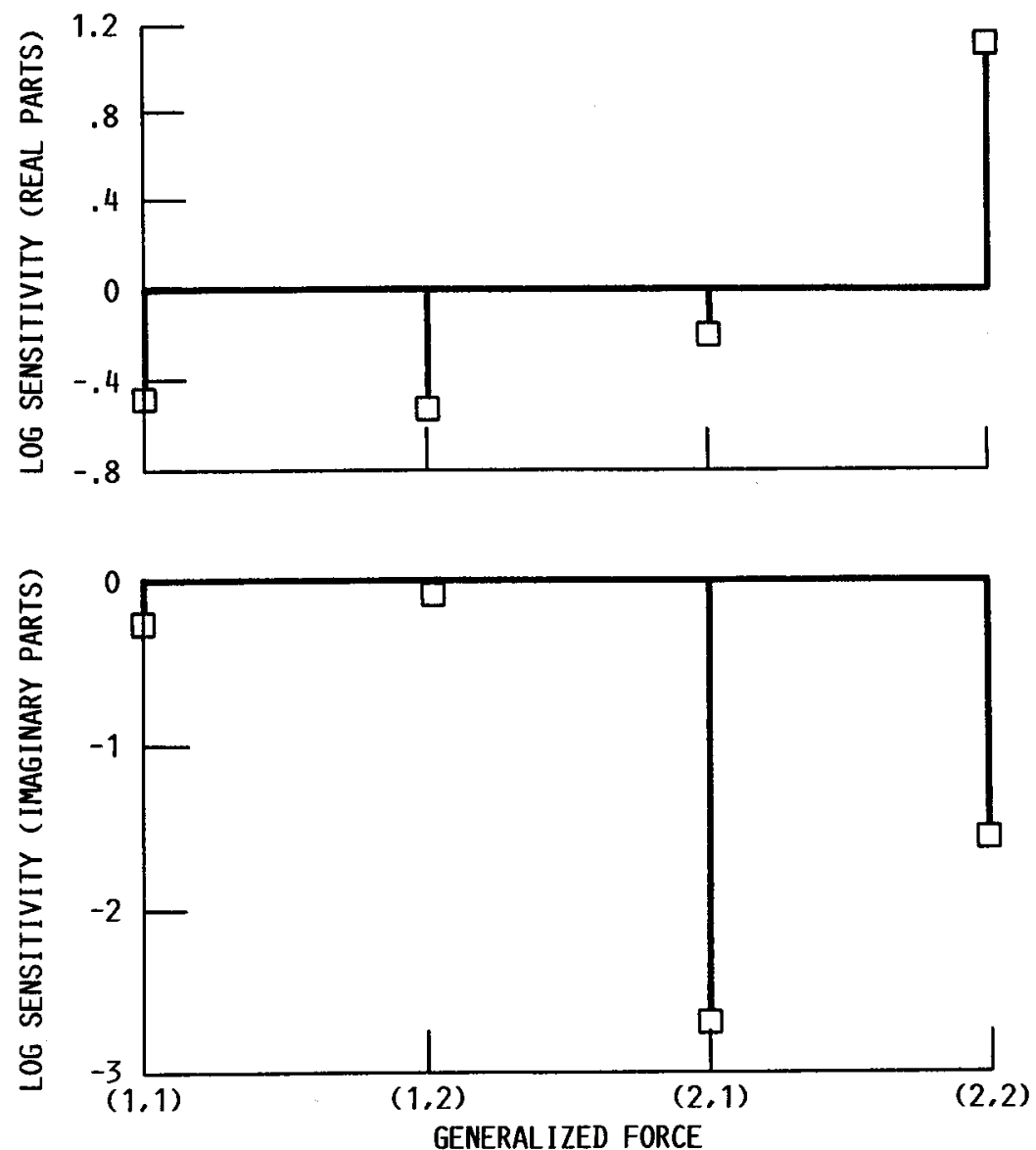

FIGURE 8. - SENSITIVITY OF GENERAL IZED FORCES TO CONTROL POINT LOCATION. 


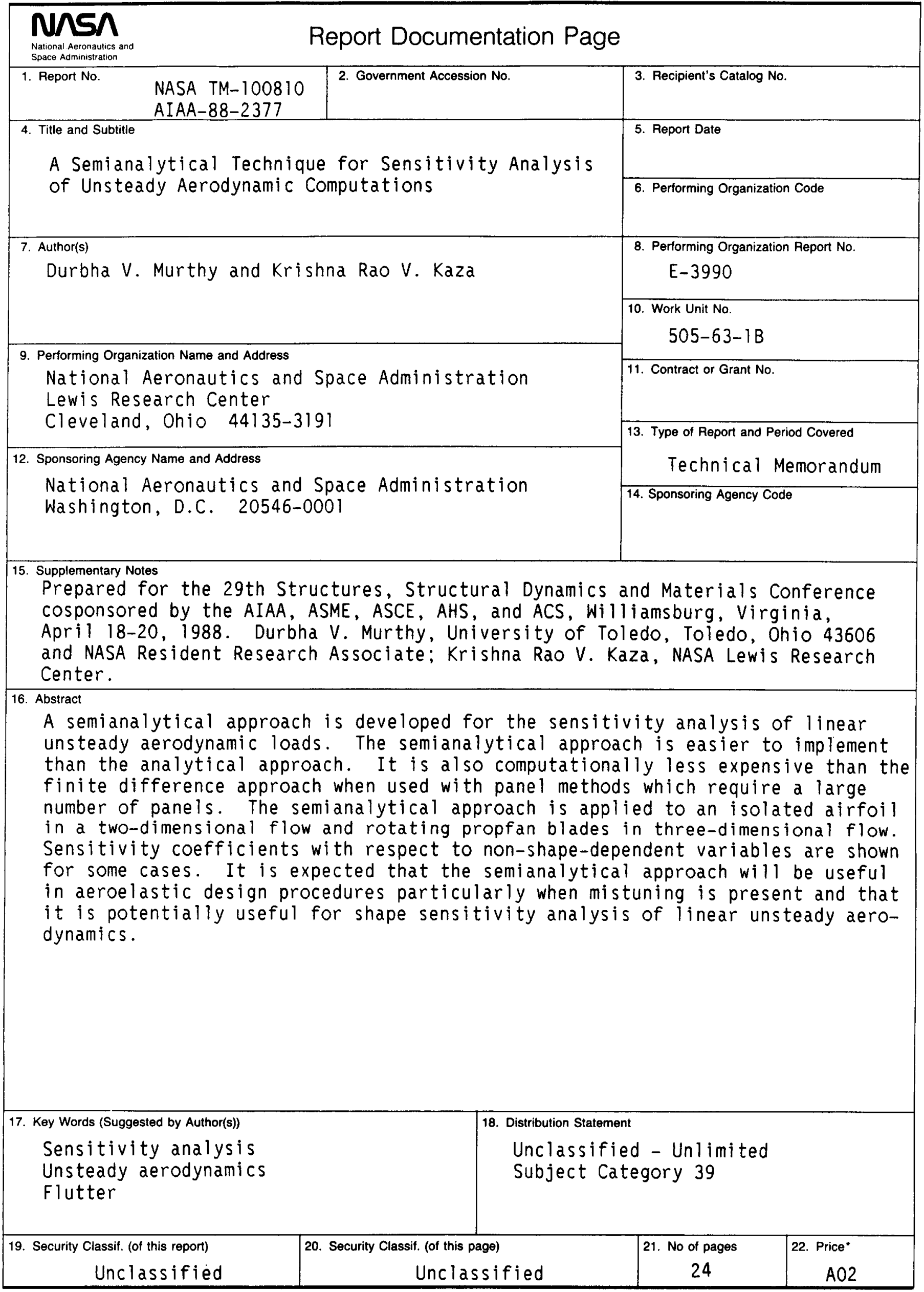

